\title{
Using Cycles and Quasi-Cycles to Disambiguate Dictionary Glosses
}

\author{
Roberto Navigli \\ Dipartimento di Informatica \\ Sapienza - Università di Roma \\ Via Salaria, 113 - 00198 Roma Italy \\ navigliedi.uniromal.it
}

\begin{abstract}
We present a novel graph-based algorithm for the automated disambiguation of glosses in lexical knowledge resources. A dictionary graph is built starting from senses (vertices) and explicit or implicit relations in the dictionary (edges). The approach is based on the identification of edge sequences which constitute cycles in the dictionary graph (possibly with one edge reversed) and relate a source to a target word sense. Experiments are performed on the disambiguation of ambiguous words in the glosses of WordNet and two machine-readable dictionaries.
\end{abstract}

\section{Introduction}

In the last two decades, we have witnessed an increasing availability of wide-coverage lexical knowledge resources in electronic format, most notably thesauri (such as Roget's Thesaurus (Roget, 1911), the Macquarie Thesaurus (Bernard, 1986), etc.), machine-readable dictionaries (e.g., the Longman Dictionary of Contemporary English (Proctor, 1978)), computational lexicons (e.g. WordNet (Fellbaum, 1998)), etc.

The information contained in such resources comprises (depending on their kind) sense inventories, paradigmatic relations (e.g. flesh ${ }_{n}^{3}$ is a kind of plant tissue $\left.{ }_{n}^{1}\right),{ }^{1}$ text definitions (e.g. flesh ${ }_{n}^{3}$ is defined as "a soft moist part of a fruit"), usage examples, and so on.

Unfortunately, not all the semantics are made explicit within lexical resources. Even WordNet, the most widespread computational lexicon of English, provides explanatory information in the form of textual glosses, i.e. strings of text

\footnotetext{
${ }^{1}$ We denote as $w_{p}^{i}$ the $i$ th sense in a reference dictionary of a word $w$ with part of speech $p$.
}

which explain the meaning of concepts in terms of possibly ambiguous words.

Moreover, while computational lexicons like WordNet contain semantically explicit information such as, among others, hypernymy and meronymy relations, most thesauri, glossaries, and machine-readable dictionaries are often just electronic transcriptions of their paper counterparts. As a result, for each entry (e.g. a word sense or thesaurus entry) they mostly provide implicit information in the form of free text.

The production of semantically richer lexical resources can help alleviate the knowledge acquisition bottleneck and potentially enable advanced Natural Language Processing applications (Cuadros and Rigau, 2006). However, in order to reduce the high cost of manual annotation (Edmonds, 2000), and to avoid the repetition of this effort for each knowledge resource, this task must be supported by wide-coverage automated techniques which do not rely on the specific resource at hand.

In this paper, we aim to make explicit large quantities of semantic information implicitly contained in the glosses of existing widecoverage lexical knowledge resources (specifically, machine-readable dictionaries and computational lexicons). To this end, we present a method for Gloss Word Sense Disambiguation (WSD), called the Cycles and Quasi-Cycles (CQC) algorithm. The algorithm is based on a novel notion of cycles in the dictionary graph (possibly with one edge reversed) which support a disambiguation choice. First, a dictionary graph is built from the input lexical knowledge resource. Next, the method explicitly disambiguates the information associated with sense entries (i.e. gloss words) by associating senses for which the richest sets of paths can be found in the dictionary graph.

In Section 2, we provide basic definitions, present the gloss disambiguation algorithm, and il- 
lustrate the approach with an example. In Section 3 , we present a set of experiments performed on a variety of lexical knowledge resources, namely WordNet and two machine-readable dictionaries. Results are discussed in Section 4, and related work is presented in Section 5. We give our conclusions in Section 6.

\section{Approach}

\subsection{Definitions}

Given a dictionary $D$, we define a dictionary graph as a directed graph $G=(V, E)$ whose vertices $V$ are the word senses in the sense inventory of $D$ and whose set of unlabeled edges $E$ is obtained as follows:

i) Initially, $E:=\emptyset$;

ii) For each sense $s \in V$, and for each lexicosemantic relation in $D$ connecting sense $s$ to $s^{\prime} \in V$, we perform: $E:=E \cup\left\{\left(s, s^{\prime}\right)\right\}$;

iii) For each sense $s \in V$, let $g \operatorname{los}(s)$ be the set of content words in its part-of-speech tagged gloss. Then for each content word $w^{\prime}$ in $g \operatorname{loss}(s)$ and for each sense $s^{\prime}$ of $w^{\prime}$, we add the corresponding edge to the dictionary graph, i.e.: $E:=E \cup\left\{\left(s, s^{\prime}\right)\right\}$.

For instance, consider WordNet as our input dictionary $D$. As a result of step (ii), given the semantic relation "sport ${ }_{n}^{1}$ is a hypernym of racing $_{n}^{1}$ ", the edge ( racing $\left._{n}^{1}, \operatorname{sport}_{n}^{1}\right)$ is added to $E$ (similarly, an inverse edge is added due to the hyponymy relation holding between $\operatorname{sport}_{n}^{1}$ and racing $_{n}^{1}$ ). During step (iii), the gloss of racing ${ }_{n}^{1}$ "the sport of engaging in contests of speed" is part-of-speech tagged, obtaining the following set of content words: $\left\{\right.$ sport $_{n}$, engage $_{v}$, contest $_{n}$, speed $\left._{n}\right\}$. The following edges are then added to $E:\left\{\right.$ racing $_{n}^{1}$, $\left.\operatorname{sport}_{n}^{1}\right),\left(\right.$ racing $_{n}^{1}$, sport $\left._{n}^{2}\right), \ldots,\left(\right.$ racing $_{n}^{1}$, sport $\left._{n}^{6}\right)$, $\ldots,\left(\right.$ racing $_{n}^{1}$, speed $\left._{n}^{1}\right), \ldots,\left(\right.$ racing $_{n}^{1}$, speed $\left.\left._{n}^{5}\right)\right\}$. The above steps are performed for all the senses in $V$.

We now recall the definition of graph cycle. A cycle in a graph $G$ is a sequence of edges of $G$ that forms a path $v_{1} \rightarrow v_{2} \rightarrow \cdots \rightarrow v_{n}\left(v_{i} \in V\right)$ such that the first vertex of the path corresponds to the last, i.e. $v_{1}=v_{n}$ (Cormen et al., 1990, p. 88). For example, the cycle in Figure 1(a) is given by the path racing $_{n}^{1} \rightarrow$ contest $_{n}^{1} \rightarrow$ race $_{n}^{3} \rightarrow$ run $_{n}^{3} \rightarrow$ racing $_{n}^{1}$ in the WordNet dictionary graph. In fact
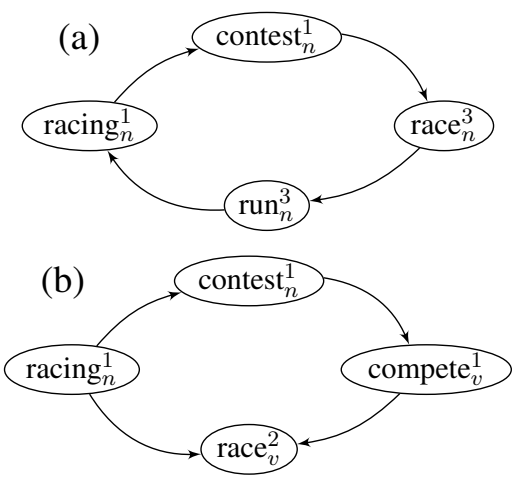

Figure 1: An example of cycle (a) and quasi-cycle (b) in WordNet.

contest $_{n}$ occurs in the gloss of racing ${ }_{n}^{1}$, race $_{n}^{3}$ is a hyponym of contest ${ }_{n}^{1}$, and so on.

We further provide the definition of quasi-cycle as a sequence of edges in which the reversal of the orientation of a single edge creates a cycle (Bohman and Thoma, 2000). For instance, the quasi-cycle in Figure 1(b) is given by the path $\mathrm{rac}$ ing $_{n}^{1} \rightarrow$ contest $_{n}^{1} \rightarrow$ compet $_{v}^{1} \rightarrow$ race $_{v}^{2} \leftarrow$ racing ${ }_{n}^{1}$. In fact, the reversal of the edge (racing ${ }_{n}^{1}$, $\operatorname{race}_{v}^{2}$ ) creates a cycle.

Finally, we call a path a (quasi-)cycle if it is either a cycle or a quasi-cycle. Further, we say that a path is (quasi-)cyclic if it forms a (quasi-)cycle in the graph.

\subsection{The CQC Algorithm}

Given a dictionary graph $G=(V, E)$ built as described in the previous section, our objective is to disambiguate dictionary glosses with the support of (quasi-)cycles. (Quasi-)cyclic paths are intuitively better than unconstrained paths as each sense choice $s$ is reinforced by the very fact of $s$ being reachable from itself through a sequence of other senses.

Let $a(s)$ be the set of ambiguous words to be disambiguated in the part-of-speech tagged gloss of sense $s$. Given a word $w^{\prime} \in a(s)$, our aim is to disambiguate $w^{\prime}$ according to the sense inventory of $D$, i.e. to assign it the right sense chosen from its set of senses Senses $\left(w^{\prime}\right)$. To this end, we propose the use of a graph-based algorithm which searches the dictionary graph and collects the following kinds of (quasi-)cyclic paths:

i) $s \rightarrow s^{\prime} \rightarrow s_{1} \rightarrow \cdots \rightarrow s_{n-2} \rightarrow s$ (cycle)

ii) $s \rightarrow s^{\prime} \rightarrow s_{1} \rightarrow \cdots \rightarrow s_{n-2} \leftarrow s$

(quasi-cycle) 


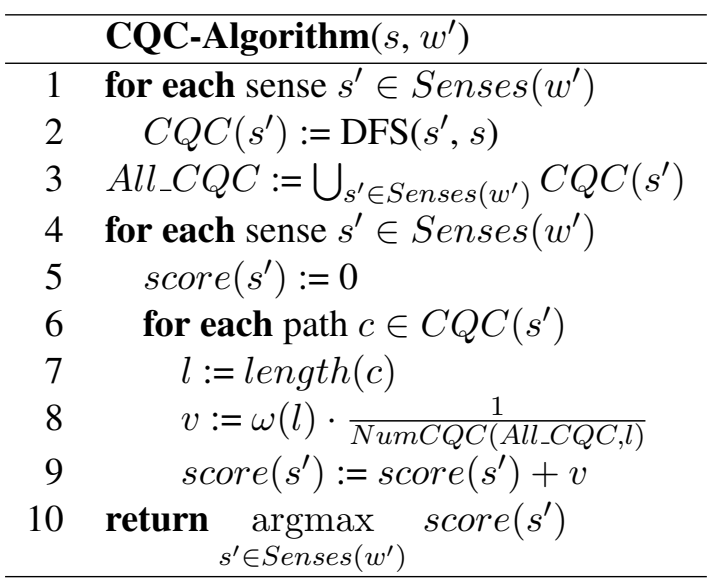

Table 1: The Cycles and Quasi-Cycles (CQC) algorithm in pseudocode.

where $s$ is our source sense, $s^{\prime}$ is a candidate sense of $w^{\prime} \in \operatorname{gloss}(s), s_{i}$ is a sense in $V$, and $n$ is the length of the path (given by the number of its edges). We note that both kinds of paths start and end with the same vertex $s$, and that we restrict quasi-cycles to those whose inverted edge departs from $s$. To avoid any redundancy, we require that no vertex is repeated in the path aside from the start/end vertex (i.e. $s \neq s^{\prime} \neq s_{i} \neq s_{j}$ for any $i, j \in\{1, \ldots, n-2\})$.

The Cycles and Quasi-Cycles (CQC) algorithm, reported in pseudo-code in Table 1, takes as input a source sense $s$ and a target word $w^{\prime}$ (in our setting ${ }^{2}$ $\left.w^{\prime} \in a(s)\right)$. It consists of two main phases.

During steps 1-3, cycles and quasi-cycles are sought for each sense of $w^{\prime}$. This step is performed with a depth-first search (DFS, cf. (Cormen et al., 1990, pp. 477-479)) up to a depth $\delta$. To this end, we first define $n \operatorname{ext}(s)=\left\{s^{\prime \prime}\right.$ : $\left.\left(s, s^{\prime \prime}\right) \in E\right\}$, that is the set of senses which can be directly reached from sense $s$. The DFS starts from a sense $s^{\prime} \in \operatorname{Senses}\left(w^{\prime}\right)$, and recursively explores the senses in next $\left(s^{\prime}\right)$ until sense $s$ or a sense in $\operatorname{next}(s)$ is encountered, obtaining a cycle or a quasi-cycle, respectively. For each sense $s^{\prime}$ of $w^{\prime}$ the DFS returns the full set $C Q C\left(s^{\prime}\right)$ of (quasi-)cyclic paths collected. Note that the DFS recursively keeps track of previously visited senses, so as to discard (quasi-)cycles including the same sense twice. Finally, in step 3, All_CQC is set to store the cycles and quasi-cycles for all the senses of $w^{\prime}$.

\footnotetext{
${ }^{2}$ Note that potentially $w^{\prime}$ can be any word of interest. The very same algorithm can be applied to determine semantic similarity or to disambiguate collocations.
}

The second phase (steps 4-10) computes a score for each sense $s^{\prime}$ of $w^{\prime}$ based on the paths collected for $s^{\prime}$ during the first phase. Let $c$ be such a path, and let $l$ be its length, i.e. the number of edges in the path. Then the contribution of $c$ to the score of $s^{\prime}$ is given by a function of its length $\omega(l)$, which associates with $l$ a number between 0 and 1 . This contribution is normalized by a factor given by $N u m C Q C\left(A l l_{-} C Q C, l\right)$, which calculates the overall number of paths of length $l$. In this work, we will employ the function $\omega(l)=1 / e^{l}$, which weighs a path with the inverse of the exponential of its length (so as to exponentially decrease the contribution of longer paths) ${ }^{3}$. Steps 4-9 are repeated for each candidate sense of $w^{\prime}$. Finally, step 10 returns the highest-scoring sense of $w^{\prime}$.

As a result of the systematic application of the CQC algorithm to the dictionary graph $G=$ $(V, E)$ associated with a dictionary $D$, a graph $\hat{G}=(V, \hat{E})$ is output, where $V$ is again the sense inventory of $D$, and $\hat{E} \subseteq E$, such that each edge $\left(s, s^{\prime}\right) \in \hat{E}$ either represents an unambiguous relation in $E$ (i.e. it was either a lexico-semantic relation in $D$ or a relation between $s$ and a monosemous word occurring in its gloss) or is the result of an execution of the CQC algorithm with input $s$ and $w^{\prime} \in a(s)$.

\subsection{An Example}

Consider the following example: WordNet defines the third sense of $f l e s h_{n}$ as "a soft moist part of a fruit". As a result of part-of-speech tagging, we obtain:

$$
\operatorname{gloss}\left(\text { flesh }_{n}^{3}\right)=\left\{\text { soft }_{a}, \text { moist }_{a}, \text { part }_{n}, \text { fruit }_{n}\right\}
$$

Let us assume we aim to disambiguate the noun fruit. Our call to the CQC algorithm in Table 1 is then CQC-Algorithm(flesh ${ }_{n}^{3}$, rruit $_{n}$ ).

As a result of the first two steps of the algorithm, a set of cycles and quasi-cycles for each sense of fruit $_{n}$ is collected, based on a DFS starting from the respective senses of our target word (we assume $\delta=5$ ). In Figure 2, we show some of the (quasi-)cycles collected for senses \#1 and \#3 of fruit $_{n}$, respectively defined as "the ripened reproductive body of a seed plant" and "an amount of a product" (we neglect sense \#2 as the length and number of its paths is not dissimilar from that of sense \#3).

\footnotetext{
${ }^{3}$ Other weight functions, such as $\omega(l)=1$ (which weighs each path independent of its length) proved to perform worse.
} 

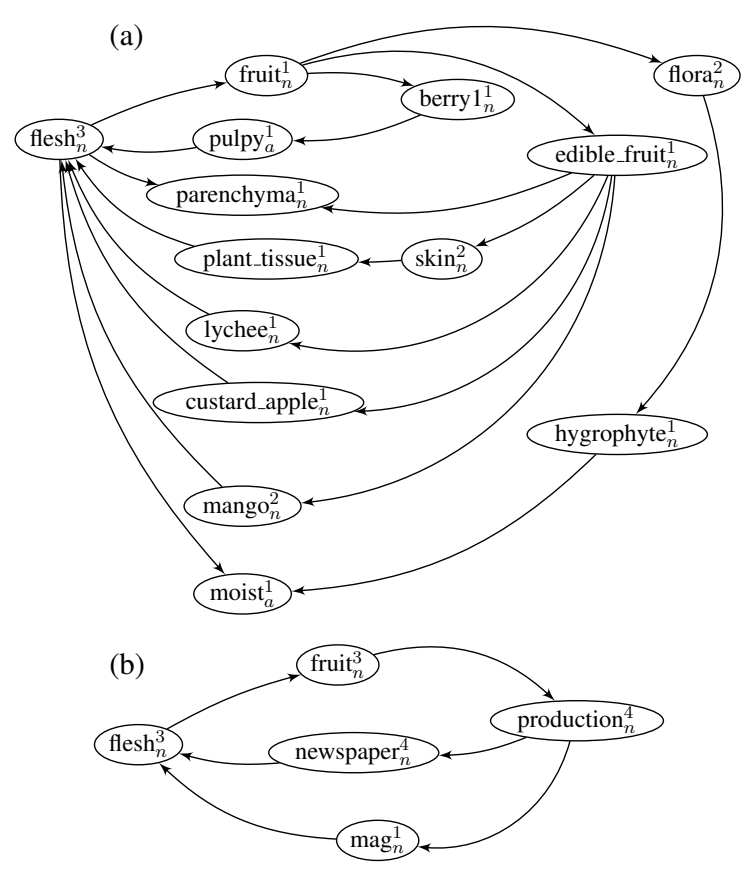

Figure 2: Some cycles and quasi-cycles connecting flesh $_{n}^{3}$ to fruit $_{n}^{1}(\mathrm{a})$, and fruit ${ }_{n}^{3}$ (b).

During the second phase of the algorithm, and for each sense of fruit $_{n}$, the contribution of each (quasi-)cycle is calculated (steps 6-9 of the algorithm). For example, for sense fruit $_{n}^{1}$ in Figure 2(a), 5 (quasi-)cycles of length 4 and 2 of length 5 were returned by DFS $\left(\right.$ fruit $_{n}^{1}$, fles $_{n}^{3}$ ). As a result, the following score is calculated: ${ }^{4}$

$$
\begin{aligned}
& \text { score }\left(\text { fruit }_{n}^{1}\right)=\frac{5}{e^{4}} \cdot \frac{1}{\text { NumCQC(all_chains }, 4)} \\
& +\frac{2}{e^{5}} \cdot \frac{1}{N u m C Q C(\text { all_chains }, 5)} \\
& =\frac{5}{e^{4.7}}+\frac{2}{e^{5.2}} \\
& =0.013+0.006=0.019
\end{aligned}
$$

whereas for fruit $_{n}^{3}$ (see Figure 2(b)) we get:

$$
\begin{aligned}
\text { score }\left(\text { fruit }_{n}^{3}\right) & =\frac{2}{e^{4}} \cdot \frac{1}{\text { NumCQC(all_chains }, 4)} \\
& =\frac{2}{e^{4} \cdot 7}=0.005
\end{aligned}
$$

where $N u m C Q C\left(A l l_{-} C Q C, l\right)$ is the total number of cycles and quasi-cycles of length $l$ over all the senses of rruit $_{n}$ (according to Figure 2, this amounts to 7 paths for $l=4$ and 2 paths for $l=5$ ).

Finally, the sense with the highest score (i.e. fruit $_{n}^{1}$ ) is returned.

\section{Experiments}

To test and compare the performance of our algorithm, we performed a set of experiments on a

\footnotetext{
${ }^{4}$ Note that, for the sake of simplicity, we are calculating our scores based on the paths shown in Figure 2. However, we tried to respect the proportion of paths collected by the algorithm for the two senses.
}

variety of resources. First, we summarize the resources (Section 3.1) and algorithms (Section 3.2) that we adopted. In Section 3.3 we report our experimental results.

\subsection{Resources}

The following resources were used in our experiments:

- WordNet (Fellbaum, 1998), the most widespread computational lexicon of English. It encodes concepts as synsets, and provides textual glosses and lexico-semantic relations between synsets. Its latest version (3.0) contains around 155,000 lemmas, and over 200,000 word senses;

- Macquarie Concise Dictionary (Yallop, 2006), a machine-readable dictionary of (Australian) English, which includes around 50,000 lemmas and almost 120,000 word senses, for which it provides textual glosses and examples;

- Ragazzini/Biagi Concise (Ragazzini and Biagi, 2006), a bilingual English-Italian dictionary, containing over 90,000 lemmas and 150,000 word senses. The dictionary provides Italian translations for each English word sense, and vice versa.

We used TreeTagger (Schmid, 1997) to part-ofspeech tag the glosses in the three resources.

\subsection{Algorithms}

Hereafter we briefly summarize the algorithms that we applied in our experiments:

- CQC: we applied the CQC algorithm as described in Section 2.2;

- Cycles, which applies the CQC algorithm but searches for cycles only (i.e. quasi-cycles are not collected);

- An adaptation of the Lesk algorithm (Lesk, 1986), which, given a source sense $s$ of word $w$ and a word $w^{\prime}$ occurring in the gloss of $s$, determines the right sense of $w^{\prime}$ as that which maximizes the (normalized) overlap between each sense $s^{\prime}$ of $w^{\prime}$ and $s$ :

$$
\underset{s^{\prime} \in \operatorname{Senses}\left(w^{\prime}\right)}{\operatorname{argmax}} \frac{\left|\operatorname{next}^{*}(s) \cap n \operatorname{ext}^{*}\left(s^{\prime}\right)\right|}{\max \left\{\left|n \operatorname{ext}^{*}(s)\right|,\left|n \operatorname{ext}^{*}\left(s^{\prime}\right)\right|\right\}}
$$


where we define $\operatorname{next}^{*}(s)=\operatorname{words}(s) \cup$ $\operatorname{next}(s)$, and words $(s)$ is the set of lexicalizations of sense $s$ (e.g. the synonyms in the synset $s$ ). When WordNet is our reference resource, we employ an extension of the Lesk algorithm, namely Extended Gloss Overlap (Banerjee and Pedersen, 2003), which extends the sense definition with words from the definitions of related senses (such as hypernyms, hyponyms, etc.). We use the same set of relations available in the authors' implementation of the algorithm.

We also compared the performance of the above algorithms with two standard baselines, namely the First Sense Baseline (abbreviated as FS BL) and the Random Baseline (Random BL).

\subsection{Results}

Our experiments concerned the disambiguation of the gloss words in three datasets, one for each resource, namely WordNet, Macquarie Concise, and Ragazzini/Biagi. In all datasets, given a sense $s$, our set $a(s)$ is given by the set of part-of-speechtagged ambiguous content words in the gloss of sense $s$ from our reference dictionary.

WordNet. When using WordNet as a reference resource, given a sense $s$ whose gloss we aim to disambiguate, the dictionary graph includes not only edges connecting $s$ to senses of gloss words (step (iii) of the graph construction procedure, cf. Section 2.1), but also those obtained from any of the WordNet lexico-semantics relations (step (ii)).

For WordNet gloss disambiguation, we employed the dataset used in the Senseval-3 Gloss WSD task (Litkowski, 2004), which contains 15,179 content words from 9,257 glosses $^{5}$. We compared the performance of CQC, Cycles, Lesk, and the two baselines. To get full coverage and high performance, we learned a threshold for each system below which they recur to the FS heuristic. The threshold and maximum path length were tuned on a small in-house manually-annotated dataset of 100 glosses. The results are shown in Table 2. We also included in the table the performance of the best-ranking system in the Senseval-

\footnotetext{
${ }^{5}$ Recently, Princeton University released a richer corpus of disambiguated glosses, namely the "Princeton WordNet Gloss Corpus" (http://wordnet.princeton.edu). However, in order to allow for a comparison with the state of the art (see below), we decided to adopt the Senseval-3 dataset.
}

\begin{tabular}{|l|c|}
\hline Algorithm & Prec./Recall \\
\hline \hline CQC & 64.25 \\
Cycles & 63.74 \\
Lesk & 51.75 \\
\hline \hline TALP & $68.60 / 68.30$ \\
\hline \hline FS BL & 55.44 \\
Random BL & 26.29 \\
\hline
\end{tabular}

Table 2: Gloss WSD performance on WordNet.

3 Gloss WSD task, namely the TALP system (Castillo et al., 2004).

CQC outperforms all other proposed approaches, obtaining a $64.25 \%$ precision and recall. We note that Cycles also gets high performance, compared to Lesk and the baselines. Also, compared to CQC, the difference is not statistically significant. However, we observe that, if we do not recur to the first sense as a backoff strategy, we get a much lower recall for Cycles $(\mathrm{P}=65.39, \mathrm{R}=$ 26.70 for CQC, $\mathrm{P}=72.03, \mathrm{R}=16.39$ for Cycles).

CQC performs about 4 points below the TALP system. As also discussed later, we believe this result is relevant, given that our approach does not rely on additional knowledge resources, as TALP does (though both algorithms recur to the FS backoff strategy).

Finally, we observe that the FS baseline has lower performance than in typical all-words disambiguation settings (usually above $60 \%$ accuracy). We believe that this is due to the absence of monosemous words from the test set, and to the possibly different distribution of senses in the dataset.

Macquarie Concise. Automatically disambiguating glosses in a computational lexicon such as WordNet is certainly useful. However, disambiguating a machine-readable dictionary is an even more ambitious task. In fact, while computational lexicons typically encode some explicit semantic relations which can be used as an aid to the disambiguation task, machine-readable dictionaries only rarely provide sense-tagged information (often in the form of references to other word senses). As a result, in this latter setting the dictionary graph typically contains only edges obtained from the gloss words of sense $s$ (step (iii), Section 2.1).

To experiment with machine-readable dictionaries, we employed the Macquarie Concise Dic- 


\begin{tabular}{|l|c|}
\hline Algorithm & Prec./Recall \\
\hline \hline CQC & 77.13 \\
Cycles & 67.63 \\
Lesk & 30.16 \\
\hline \hline FS BL & 51.48 \\
Random BL & 23.28 \\
\hline
\end{tabular}

Table 3: Gloss WSD performance on Macquarie Concise.

tionary (Yallop, 2006). A dataset was prepared by randomly selecting 1,000 word senses from the dictionary and annotating the content words in their glosses according to the dictionary sense inventory. Overall, 2,678 words were sense tagged.

The results are shown in Table 3. CQC obtains an accuracy of $77.13 \%$ (in case of ties, a random choice is made, thus leading to the same precision and recall), Cycles achieves an accuracy of almost $10 \%$ less than CQC (the difference is statistically significant; $p<0.01$ ). The FS baseline, here, is based on the first sense listed in the Macquarie sense inventory, which - in contrast to WordNet - does not depend on the occurrence frequency of senses in a semantically-annotated corpus. However, we note that the FS baseline is not very different from that of the WordNet experiment.

We observe that the Lesk performance is very low on this dataset (around 7 points above the Random BL), due to the impossibility of using the Extended Gloss Overlap approach (semantic relations are not available in the Macquarie Concise) and to the low number of matches between source and target entries.

Ragazzini/Biagi. Finally, we performed an experiment on the Ragazzini/Biagi English-Italian machine-readable dictionary. In this experiment, disambiguating a word $w^{\prime}$ in the gloss of a sense $s$ from one section (e.g. Italian-English) equals to selecting a word sense $s^{\prime}$ of $w^{\prime}$ listed in the other section of the dictionary (e.g. English-Italian). For example, given the English entry race $e_{n}^{1}$, translated as "corsa ${ }_{n}, \operatorname{gara}_{n}$ ", our objective is to assign the right Italian sense from the Italian-English section to $\operatorname{corsa}_{n}$ and $\operatorname{gara}_{n}$.

To apply the CQC algorithm, a simple adaptation is needed, so as to allow (quasi-)cycles to connect word senses from the two distinct sections. The algorithm must seek cyclic and quasi-cyclic paths, respectively of the kind:

\begin{tabular}{|l|c|}
\hline Algorithm & Prec./Recall \\
\hline \hline CQC & 89.34 \\
Cycles & 85.40 \\
Lesk & 63.89 \\
\hline \hline FS BL & 73.15 \\
Random BL & 51.69 \\
\hline
\end{tabular}

Table 4: Gloss WSD performance on Ragazzini/Biagi.

$$
\begin{aligned}
& \text { i) } s \rightarrow s^{\prime} \rightarrow s_{1} \rightarrow \cdots \rightarrow s_{n-2} \rightarrow s \\
& \text { ii) } s \rightarrow s^{\prime} \rightarrow s_{1} \rightarrow \cdots \rightarrow s_{n-2} \leftarrow s
\end{aligned}
$$

where $n$ is the path length, $s$ and $s^{\prime}$ are senses respectively from the source (e.g. Italian/English) and the target (e.g. English/Italian) section of the dictionary, $s_{i}$ is a sense from the target section for $i \leq k$ and from the source section for $i>k$, for some $k$ such that $0 \leq k \leq n-2$. In other words, the DFS can jump at any time from the target section to the source section. After the jump, the depth search continues in the source section, in the hope to reach $s$. For example, the following is a cycle with $k=1$ :

$$
\operatorname{race}_{n}^{1} \rightarrow \operatorname{corsa}_{n}^{2} \rightarrow \operatorname{gara}_{n}^{2} \rightarrow \operatorname{race}_{n}^{1}
$$

where the edge between $\operatorname{cors} a_{n}^{2}$ and $g a r a_{n}^{2}$ is due to the occurrence of gara $_{n}$ in the gloss of $\operatorname{cors} a_{n}^{2}$ as a domain label for that sense.

To perform this experiment, we randomly selected 250 entries from each section (500 overall), including a total number of 1,069 translations that we manually sense tagged. In Table 4 we report the results of CQC, Cycles and Lesk on this task. Overall, the figures are higher than in previous experiments, thanks to a lower average degree of polysemy of the resource, which also impacts positively on the FS baseline. However, given a random baseline of $51.69 \%$, the performance of CQC, over $89 \%$ precision and recall, is significantly higher. Cycles obtains around 4 points less than CQC (the difference is statistically significant; $p<0.01$ ). The performance of Lesk $(63.89 \%)$ is also much higher than in our previous experiments, thanks to the higher chance of finding a 1:1 correspondence between the two sections. However, we observed that this does not always hold, as also supported by the better results of CQC. 


\section{Discussion}

The experiments presented in the previous section are inherently heterogeneous, due to the different nature of the resources adopted (a computational lexicon, a monolingual and a bilingual machinereadable dictionary). Our aim was to show the flexibility of our approach in tagging gloss words with senses from the same dictionary.

We show the average polysemy of the three datasets in Table 5. Notice that none of the datasets included monosemous items, so our experiments cannot be compared to typical all-words disambiguation tasks, where monosemous words are part of the test set.

Given that words in the Macquarie dataset have a higher average polysemy than in the WordNet dataset, one might wonder why disambiguating glosses from a computational lexicon such as WordNet is more difficult than performing a similar task on a machine-readable dictionary such as the Macquarie Concise Dictionary, which does not provide any explicit semantic hint. We believe there are at least two reasons for this outcome: the first specifically concerns the Senseval3 Gloss WSD dataset, which does not reflect the distribution of genus-differentiae terms in dictionary glosses: less than $10 \%$ of the items were hypernyms, thus making the task harder. As for the second reason, we believe that the Macquarie Concise provides more clear-cut definitions, thus making sense assignments relatively easier.

An analytical comparison of the results of $\mathrm{Cy}$ cles and CQC show that, especially for machinereadable dictionaries, employing both cycles and quasi-cycles is highly beneficial, as additional support is provided by the latter patterns. Our results on WordNet prove to be more difficult to analyze, because of the need of employing the first sense heuristic to get full coverage. Also, the maximum path length used for WordNet was different $(\delta=3$ according to our tuning, compared to $\delta=4$ for Macquarie and Ragazzini/Biagi). However, quasicycles are shown to provide over $10 \%$ improvement in terms of recall (at the price of a decrease in precision of 6.6 points).

Further, we note that the performance of the CQC algorithm dramatically improves as the maximum score (i.e. the score which leads to a sense assignment) increases. As a result, users can tune the disambiguation performance based on their specific needs (coverage, precision, etc.). For in-

\begin{tabular}{|l|c|c|c|}
\hline & WN & Mac & R/B \\
\hline Polysemy & 6.68 & 7.97 & 3.16 \\
\hline
\end{tabular}

Table 5: Average polysemy of the three datasets.

stance, WordNet Gloss WSD can perform up to $85.7 \%$ precision and $10.1 \%$ recall if we require the score to be $\geq 0.2$ and do not use the FS baseline as a backoff strategy. Similarly, we can reach up to 93.8\% prec., 20.0\% recall for Macquarie Concise (score $\geq 0.12$ ) and even $95.2 \%$ prec., $70.6 \%$ recall (score $\geq 0.1$ ) for Ragazzini/Biagi.

\section{Related Work}

Word Sense Disambiguation is a large research field (see (Navigli, 2009) for an up-to-date overview). However, in this paper we focused on a specific kind of WSD, namely the disambiguation of dictionary definitions. Seminal works on the topic date back to the late 1970s, with the development of models for the identification of taxonomies from lexical resources (Litkowski, 1978; Amsler, 1980). Subsequent works focused on the identification of genus terms (Chodorow et al., 1985) and, more in general, on the extraction of explicit information from machine-readable dictionaries (see, e.g., (Nakamura and Nagao, 1988; Ide and Véronis, 1993)). Kozima and Furugori (1993) provide an approach to the construction of ambiguous semantic networks from glosses in the Longman Dictionary of Contemporary English (LDOCE). In this direction, it is worth citing the work of Vanderwende (1996) and Richardson et al. (1998), who describe the construction of MindNet, a lexical knowledge base obtained from the automated extraction of lexico-semantic information from two machine-readable dictionaries. As a result, weighted relation paths are produced to infer the semantic similarity between pairs of words.

Several heuristics have been presented for the disambiguation of the genus of a dictionary definition (Wilks et al., 1996; Rigau et al., 1997). More recently, a set of heuristic techniques has been proposed to semantically annotate WordNet glosses, leading to the release of the eXtended WordNet (Harabagiu et al., 1999; Moldovan and Novischi, 2004). Among the methods, the cross reference heuristic is the closest technique to our notion of cycles and quasi-cycles. Given a pair of words $w$ and $w^{\prime}$, this heuristic is based on the occurrence of 
$w$ in the gloss of a sense $s^{\prime}$ of $w^{\prime}$ and, vice versa, of $w^{\prime}$ in the gloss of a sense $s$ of $w$. In other words, a graph cycle $s \rightarrow s^{\prime} \rightarrow s$ of length 2 is sought.

Based on the eXtended WordNet, a gloss disambiguation task was organized at Senseval-3 (Litkowski, 2004). Interestingly, the best performing systems, namely the TALP system (Castillo et al., 2004), and SSI (Navigli and Velardi, 2005), are knowledge-based and rely on rich knowledge resources: respectively, the Multilingual Central Repository (Atserias et al., 2004), and a proprietary lexical knowledge base.

In contrast, the approach presented in this paper performs the disambiguation of ambiguous words by exploiting only the reference dictionary itself. Furthermore, as we showed in Section 3.3, our method does not rely on WordNet, and can be applied to any lexical knowledge resource, including bilingual dictionaries.

Finally, methods in the literature more focused on a specific disambiguation task include statistical methods for the attachment of hyponyms under the most likely hypernym in the WordNet taxonomy (Snow et al., 2006), structural approaches based on semantic clusters and distance metrics (Pennacchiotti and Pantel, 2006), supervised machine learning methods for the disambiguation of meronymy relations (Girju et al., 2003), etc.

\section{Conclusions}

In this paper we presented a novel approach to disambiguate the glosses of computational lexicons and machine-readable dictionaries, with the aim of alleviating the knowledge acquisition bottleneck. The method is based on the identification of cycles and quasi-cycles, i.e. circular edge sequences (possibly with one edge reversed) relating a source to a target word sense.

The strength of the approach lies in its weakly supervised nature: (quasi-)cycles rely exclusively on the structure of the input lexical resources. No additional resource (such as labeled corpora or external knowledge bases) is required, assuming we do not resort to the FS baseline. As a result, the approach can be applied to obtain a semantic network from the disambiguation of virtually any lexical resource available in machine-readable format for which a sense inventory is provided.

The utility of gloss disambiguation is even greater in bilingual dictionaries, as idiosyncrasies such as missing or redundant translations can be discovered, thus helping lexicographers improve the resources ${ }^{6}$. An adaptation similar to that described for disambiguating the Ragazzini/Biagi can be employed for mapping pairs of lexical resources (e.g. FrameNet (Baker et al., 1998) to WordNet), thus contributing to the beneficial knowledge integration process. Following this direction, we are planning to further experiment on the mapping of FrameNet, VerbNet (Kipper et al., 2000), and other lexical resources.

The graphs output by the CQC algorithm for our datasets are available from http://lcl.uniromal.it/cqc. We are scheduling the release of a software package which includes our implementation of the CQC algorithm and allows its application to any resource for which a standard interface can be written.

Finally, starting from the work of Budanitsky and Hirst (2006), we plan to experiment with the CQC algorithm when employed as a semantic similarity measure, and compare it with the most successful existing approaches. Although in this paper we focused on the disambiguation of dictionary glosses, the same approach can be applied for disambiguating collocations according to a dictionary of choice, thus providing a way to further enrich lexical resources with external knowledge.

\section{Acknowledgments}

The author is grateful to Ken Litkowski and the anonymous reviewers for their useful comments. He also wishes to thank Zanichelli and Macquarie for kindly making their dictionaries available for research purposes.

\section{References}

Robert A. Amsler. 1980. The structure of the Merriam-Webster pocket dictionary, Ph.D. Thesis. University of Texas, Austin, TX, USA.

Jordi Atserias, Luís Villarejo, German Rigau, Eneko Agirre, John Carroll, Bernardo Magnini, and Piek Vossen. 2004. The meaning multilingual central repository. In Proceedings of GWC 2004, pages 2330, Brno, Czech Republic.

Collin F. Baker, Charles J. Fillmore, and John B. Lowe. 1998. The berkeley framenet project. In Proceedings of COLING-ACL 1998, pages 86-90, Montreal, Canada.

\footnotetext{
${ }^{6}$ This is indeed an ongoing line of research in collaboration with the Zanichelli dictionary publisher.
} 
Satanjeev Banerjee and Ted Pedersen. 2003. Extended gloss overlaps as a measure of semantic relatedness. In Proceedings of IJCAI 2003, pages 805-810, Acapulco, Mexico.

John Bernard, editor. 1986. Macquarie Thesaurus. Macquarie, Sydney, Australia.

Tom Bohman and Lubos Thoma. 2000. A note on sparse random graphs and cover graphs. The Electronic Journal of Combinatorics, 7:1-9.

Alexander Budanitsky and Graeme Hirst. 2006. Evaluating wordnet-based measures of semantic distance. Computational Linguistics, 32(1):13-47.

Mauro Castillo, Francis Real, Jordi Asterias, and German Rigau. 2004. The talp systems for disambiguating wordnet glosses. In Proceedings of ACL 2004 SENSEVAL-3 Workshop, pages 93-96, Barcelona, Spain.

Martin Chodorow, Roy Byrd, and George Heidorn. 1985. Extracting semantic hierarchies from a large on-line dictionary. In Proceedings of ACL 1985, pages 299-304, Chicago, IL, USA.

Thomas H. Cormen, Charles E. Leiserson, and Ronald L. Rivest. 1990. Introduction to algorithms. MIT Press, Cambridge, MA.

Montse Cuadros and German Rigau. 2006. Quality assessment of large scale knowledge resources. In Proceedings of EMNLP 2006, pages 534-541, Sydney, Australia.

Philip Edmonds. 2000. Designing a task for SENSEVAL-2. Technical note.

Christiane Fellbaum, editor. 1998. WordNet: An Electronic Database. MIT Press, Cambridge, MA.

Roxana Girju, Adriana Badulescu, and Dan Moldovan. 2003. Learning semantic constraints for the automatic discovery of part-whole relations. In Proceedings of NAACL 2003, pages 1-8, Edmonton, Canada.

Sanda Harabagiu, George Miller, and Dan Moldovan. 1999. Wordnet 2 - a morphologically and semantically enhanced resource. In Proceedings of SIGLEX-99, pages 1-8, Maryland, USA.

Nancy Ide and Jean Véronis. 1993. Extracting knowledge bases from machine-readable dictionaries: Have we wasted our time? In Proceedings of Workshop on Knowledge Bases and Knowledge Structures, pages 257-266, Tokyo, Japan.

Karin Kipper, Hoa Trang Dang, and Martha Palmer. 2000. Class-based construction of a verb lexicon. In Proceedings of AAAI 2000, pages 691-696, Austin, TX, USA.

Hideki Kozima and Teiji Furugori. 1993. Similarity between words computed by spreading activation on an english dictionary. In Proceedings of ACL 1993, pages 232-239, Utrecht, The Netherlands.

Michael Lesk. 1986. Automatic sense disambiguation using machine readable dictionaries: How to tell a pine cone from an ice cream cone. In Proceedings of the $5^{\text {th }}$ SIGDOC, pages 24-26, New York, NY.

Kenneth C. Litkowski. 1978. Models of the semantic structure of dictionaries. American Journal of Computational Linguistics, (81):25-74.
Kenneth C. Litkowski. 2004. Senseval-3 task: Word-sense disambiguation of wordnet glosses. In Proceedings of ACL 2004 SENSEVAL-3 Workshop, pages 13-16, Barcelona, Spain.

Dan Moldovan and Adrian Novischi. 2004. Word sense disambiguation of wordnet glosses. Computer Speech \& Language, 18:301-317.

Jun-Ichi Nakamura and Makoto Nagao. 1988. Extraction of semantic information from an ordinary english dictionary and its evaluation. In Proceedings of COLING 1988, pages 459-464, Budapest, Hungary.

Roberto Navigli and Paola Velardi. 2005. Structural semantic interconnections: a knowledge-based approach to word sense disambiguation. IEEE Transactions of Pattern Analysis and Machine Intelligence (TPAMI), 27(7):1075-1088.

Roberto Navigli. 2009. Word sense disambiguation: a survey. ACM Computing Surveys, 41(2):1-69.

Marco Pennacchiotti and Patrick Pantel. 2006. Ontologizing semantic relations. In Proceedings of COLING-ACL 2006, pages 793-800, Sydney, Australia.

Paul Proctor, editor. 1978. Longman Dictionary of Contemporary English. Longman Group, UK.

Giuseppe Ragazzini and Adele Biagi, editors. 2006. Il Ragazzini-Biagi, $4^{\text {th }}$ Edition. Zanichelli, Italy.

Stephen D. Richardson, William B. Dolan, and Lucy Vanderwende. 1998. Mindnet: acquiring and structuring semantic information from text. In Proceedings of COLING 1998, pages 1098-1102, Montreal, Quebec, Canada.

German Rigau, Jordi Atserias, and Eneko Agirre. 1997. Combining unsupervised lexical knowledge methods for word sense disambiguation. In Proceedings of ACL/EACL 1997, pages 48-55, Madrid, Spain.

Peter M. Roget. 1911. Roget's International Thesaurus ( $1^{\text {st }}$ edition). Cromwell, New York, USA.

Helmut Schmid. 1997. Probabilistic part-of-speech tagging using decision trees. In Daniel Jones and Harold Somers, editors, New Methods in Language Processing, Studies in Computational Linguistics, pages 154-164. UCL Press, London, UK.

Rion Snow, Daniel Jurafsky, and Andrew Y. Ng. 2006. Semantic taxonomy induction from heterogenous evidence. In Proceedings of COLING-ACL 2006, pages 801-808, Sydney, Australia.

Lucy Vanderwende. 1996. The analysis of noun sequences using semantic information extracted from on-line dictionaries, Ph.D. Thesis. Georgetown University, Washington, USA.

Yorick Wilks, Brian Slator, and Louise Guthrie, editors. 1996. Electric words: Dictionaries, computers and meanings. MIT Press, Cambridge, MA.

Colin Yallop, editor. 2006. The Macquarie Concise Dictionary $4^{\text {th }}$ Edition. Macquarie Library Pty Ltd, Sydney, Australia. 